\title{
Acarbosa y diabetes mellitus. Implicaciones prácticas
}

Calle Pascual AL, Charro Salgado AL. Acarbosa y diabetes mellitus. Implicaciones prácticas. An Med Interna (Madrid) 2001; 18: 231-233.

La acarbosa es un fármaco oral antihiperglucemiante que produce una mejoría del control metabólico de la diabetes mellitus disminuyendo la respuesta glucémica postprandial (1-3).

Es el representante mas antiguo de la familia de los inhibidores competitivos de las alfa glucosidasas. Las alfa glucosidasas son una familia de enzimas responsables de la hidrólisis de los carbohidratos en el aparato digestivo. Estas enzimas están localizadas fundamentalmente en el borde de las vellosidades del intestino delgado, y su inhibición produce un retraso en la digestión de los carbohidratos cuya absorción se prolonga. Esto significa que la elevación postprandial de la glucemia se reduce y se prolonga, pues no produce malabsorción. El impacto que esta disminución en la glucemia postprandial tiene sobre el control metabólico de la diabetes mellitus ha sido controvertido, y aunque admitida su eficacia, no esta totalmente establecido en que momento de la historia de la diabetes mellitus, y en que tipo de diabetes debe ser empleado. Por otro lado, los efectos secundarios que produce, sobre todo la flatulencia y aerofagia ha limitado de una forma sustancial su utilización. En este número, González Sarmiento y (4) publican un estudio sobre la eficacia del tratamiento con acarbosa en pacientes diabéticos tipo 2 tratados con insulina, o sulfonilurea o con dieta solo. El análisis se realiza del grupo de pacientes en conjunto y no se controla algunas variables importantes. Sin embargo existen suficientes evidencias en la literatura sacadas de estudios controlados, doble ciego y cruzados, muchos de ellos multicéntricos, cuyas conclusiones tienen un nivel de evidencia fuerte (nivel A). Estos estudios sirven de base para realizar una recomendación generalizable a todos los pacientes con diabetes mellitus.

\section{ACARBOSA Y DIABETES TIPO 2}

La diabetes tipo 2 es una enfermedad heterogénea que engloba una serie de síndromes de origen genético o adquiridos, caracterizada por la presencia de resistencia al efecto hipoglucemiante de la insulina al que acompaña un defecto en la secreción de insulina (5). En el momento del diagnóstico, cuando aparece la hiperglucemia, ambos factores están presentes, aunque predomine la resistencia a la insulina en la mayoría de los casos. En la evolución de la diabetes tipo 2, el defecto en la secreción de insulina es cada vez mas pronunciado, haciendo que la mayor parte de los pacientes precisen tratamiento con insulina exógena. Por lo tanto, es necesario analizar de forma separada los efectos de la acarbosa en los diferentes estados evolutivos de la diabetes mellitus.

-Acarbosa como fármaco de primera línea. Inicialmente el tratamiento de las personas con diabetes tipo 2 debe estar basado en unas recomendaciones higiénico dietéticas, basadas en la alimentación habitual que realizan y en el estilo de vida que desean llevar dichas personas. Si no logran alcanzar los objetivos terapéuticos, en particular los niveles de HbA1c y glucemia consideradas óptimas (6) se debe comenzar el tratamiento farmacológico. ¿Qué papel tiene la acarbosa en este estadio? Existen tres estudios que soportan una evidencia cuya fuerza tiene el máximo nivel (7-9). Meneilly y cols. (7) estudian 45 pacientes diabéticos tipo 2 con fracaso a tratamiento dietético. Los pacientes que recibieron acarbosa en relación con los que recibieron placebo mostraron una reducción media en la glucosa plasmática en ayunas de $13 \mathrm{mg} / \mathrm{dl}$ (10\%), y de $45 \mathrm{mg} / \mathrm{dl}$ (26\%) en la glucemia dos horas postprandial. La HbA1c tenia unos valores absolutos medios inferiores en $0,8 \%$ (descenso del 10\%), y los pacientes que recibieron acarbosa mostraron un incremento en la sensibilidad a la insulina. Hasche y cols. (8) mostraron tras dos años de seguimiento de 60 pacientes con diabetes tipo 2 y con fracaso al tratamiento nutricional un descenso en los valores de HbA1c de 0,9\% mayor en los que recibieron acarbosa en relación a los que recibieron placebo. Esto permitió que el $89 \%$ de los pacientes que recibieron acarbosa en relación al $49 \%$ de los que recibieron placebo obtuvieran un índice de respuesta considerado óptimo por el valor absoluto de HbA1c alcanzado. Nuestro grupo (9) demostró en pacientes con diabetes tipo 2, diagnosticados con sobrecarga oral de glucosa, por lo tanto en una fase clínica muy precoz, un descenso en la glucosa plasmática en ayunas de $16 \mathrm{mg} / \mathrm{dl}$, en la 
HbA1c de 0,2\%, y además un descenso modesto en el peso corporal y en la tensión arterial sistólica y diastólica, demostrando también un incremento en la sensibilidad a la insulina sin modificar la capacidad secretagoga del páncreas, en los pacientes que recibieron acarbosa en comparación con los que recibieron placebo. Ningún estudio demostró que afectara al metabolismo lipídico, aunque se observó una tendencia a reducir el nivel de triglicéridos, ni que afectara la eliminación de albuminuria.

- Acarbosa en combinación con otros fármacos. Como en la evolución de la diabetes tipo 2 se combinan los defectos en la acción y en la secreción de insulina varios estudios han sido enfocados para evaluar la eficacia de añadir acarbosa con diferentes fármacos hipoglucemiantes y en diferentes momentos evolutivos antes o después de que alcanzaran la dependencia de insulina (10-15). López Alvarenga y cols. (10) comparan los efectos que tiene añadir acarbosa o insulina en pacientes insuficientemente tratados con sulfonilureas y metformina. La acarbosa logra frente a placebo una reducción mayor en los valores de HbA1c del $10 \%$ y de la glucemia plasmática en ayunas del $15 \%$. Sin embargo esta reducción es mas importante administrando una sola dosis de insulina nocturna, alcanzando el $40 \%$ en la HbA1c y el $23 \%$ en la glucosa plasmática en ayunas. De forma semejante nuestro grupo (11) demostró que al añadir acarbosa a pacientes insuficientemente tratados con dosis máximas de sulfonilureas, se producía un reducción mas modesta en dichos valores, siendo de igual forma inferiores a las que presentaban cuando se añadía metformina o insulina. Tanto la metformina como la insulina presentaban ventajas añadidas sobre la sensibilidad a la insulina y los niveles plasmáticos de lipoproteínas. Willms y cols. (12) encontraron un comportamiento parecido entre la acarbosa y la metformina cuando se añadía a dosis máximas de sulfonilureas. Sin embargo metformina tenia ventajas sobre otros aspectos como la ganancia de peso. Meneilly y cols. (13) en unos pacientes tratados insuficientemente con dosis máximas de sulfonilureas demuestra unos resultados parecidos al añadir acarbosa. Varios estudio valoran la eficacia de añadir acarbosa en pacientes con diabetes tipo 2 tratados con insulina, es decir en una fase de evolución mas tardía, cuando existe un mayor déficit en la secreción de insulina. Standl y cols. (14) estudian los efectos de añadir acarbosa a pacientes tratados con una combinación de insulina y sulfonilureas, encontrando que acarbosa logra una reducción media de la dosis de insulina en 8 unidades, del $15 \%$ en los niveles de HbA1c y mejora la sensibilidad a la insulina. Guvenor y cols. (15) estudian los efectos de añadir sulfonilureas en comparación con acarbosa en pacientes tratados con insulina NPH y regular en dos dosis diarias. Los resultados obtenidos no demuestran ninguna ventaja sobre el control glucémico al añadir acarbosa. De interés particular resulta el estudio de Gentile y cols. (16) donde pacientes con cirrosis hepática y diabetes tipo 2 tratada con insulina se les añade acarbosa. Además de encontrar unos efectos equivalentes sobre el control glucémico, estimado en una reducción del $10 \%$ de la glucosa plasmática en ayunas y en el valor de HbA1c, obtienen una mejoría en la flora intestinal, muy importante para la prevención de la encefalopatía hepática. Cabe reseñar además, por la relevancia que para el tratamiento de la diabetes tipo 2 tiene el estudio UKPDS, que 1.946 pacientes incluidos en dicho estudio fueron tratados de forma aleatoria con acarbosa y seguidos durante 3 años (17). El tratamiento con acarbosa tan solo lo continuaron los 3 años un $39 \%$ de los pacientes, por sus efectos secundarios, fundamentalmente flatulencia y diarrea. La mediana de HbA1c descendió modestamente, $0,2 \%$, siendo la diferencia con placebo de $0,5 \%$. En este estudio no se observó diferencias en otros parámetros bioquímicos ni en la evolución de las complicaciones.

\section{ACARBOSA Y DIABETES TIPO 1}

No existen estudios controlados, aleatorios y doble ciego que demuestren la eficacia del tratamiento con acarbosa en el tratamiento de los pacientes con diabetes tipo 1. Tan sólo existen dos estudios en que se demuestra alguna ventaja, como la disminución del tiempo entre la administración de insulina y la ingesta de la comida (18) o la menor necesidad de insulina para metabolizar los alimentos ricos en carbohidratos ingeridos (19).

\section{CONCLUSIONES Y RECOMENDACIONES PRÁCTICAS}

En función de los hallazgos encontrados en estudios realizados de forma aleatoria, controlada, doble ciego en comparación con placebo, se puede concluir que la acarbosa tiene un efecto beneficioso sobre el control metabólico de la diabetes tipo 2 , que se puede resumir en lograr reducir la glucemia plasmática en ayunas entre un 10 y $15 \%$, y la HbA1c entre un 5 y $10 \%$. Si el tratamiento con acarbosa se instaura precozmente, por ejemplo en pacientes que no alcanzan un buen control glucémico con tratamiento higiénico dietético, el control glucémico puede acercarse al considerado óptimo, y además se pueden conseguir ventajas añadidas sobre la sensibilidad a la insulina. Si se instaura en fases mas avanzadas de la enfermedad, por ejemplo en pacientes con fracaso secundario a dosis máximas de hipoglucemiante oral, los efectos beneficiosos sobre el control glucémico son mas modestos, no se alcanzan los niveles considerados óptimos en dichos parámetros, y su eficacia es menor a la que se obtiene con otros tratamientos farmacológicos, por ejemplo con insulina. No existe ninguna evidencia que demuestre que afecte la evolución de las complicaciones de la diabetes. De forma semejante tampoco existe ninguna evidencia que tenga efectos beneficiosos sobre el control metabólico de la diabetes tipo 1.

Basándonos en la evidencia, la mayor indicación clínica sería recomendar su utilización en las fases iniciales del tratamiento de las personas con diabetes tipo 2. Los efectos secundarios que presenta pueden limitar su utilización.

\section{A. L. CALLE PASCUAL Y A. L. CHARRO SALGADO}

Servicio de Endocrinología y Nutrición. Hospital Clínico San Carlos. Madrid 


\section{Bibliografía}

1. Hillebrand I. Pharmacological modification of digestion and absorption. Diabe med 1987; 4: 157-150.

2. Sachse G. Acarbose in non-insulin-dependen diabetes. In Creutzfeld W. De. Acarbose for the treatment of diabetes mellitus. Berlin. Springer Verlag 1998; 92-101.

3. Coniff RF, Shapiro JA, Seaton TB, Bray GA. Multicenter, placebo-controlled trial comparing acarbose with placebo, tolbutamide and tolbutamide-plus-acarbose in non-insuñin-dependent diabetes mellitus. A multicenter controlled clinical trial. Ann Inter Med 1995; 98: 443-451.

4. González Sarmiento E, Ergueta Martín P, Fernández Martínez I, Hinojosa Mena-Bernal MC, Zurro Muñoz I, Zurro Hernández J. Modification of the metabolic parameters and micro-albuminuria in patients with diabetes mellitus type 2 treated with acarbose. An Med Interna (Madrid) 2001; 18: 234-236.

5. The expert committee on the diagnosis and classification of diabetes mellitus. Report of the expert committee on the diagnosis and classification of diabetes mellitus. Diabetes Care 2001;24 (supl 1):s5-s20.

6. American Diabetes Association (position statement). Standards of medical care for patients with diabetes mellitus. Diabetes Care 2001; 24 (supl 1):s33-43.

7. Meneilly GS, Ryan EA, Radziuk J, Lau DC, Yale JF, Morais J, Chiasson JL, Rabasa-Lhoret R, Maheux P, Tessier D, Wolever T, Josse RG, Elahi D. Effect of acarbose on insulin sensitivity in elderly patients with diabetes. Diabetes Care 2000; 23: 1162-7.

8. Hasche H, Mertes G, Bruns C, Englert R, Genthner P, Heim D, Heyen P, Mahla G, Schmidt C, Schulze-Schleppinghof B, Steger-Johannsen G Effects of acarbose treatment in Type 2 diabetic patients under dietary training: a multicentre, double-blind, placebo-controlled, 2-year study. Diabetes Nutr Metab 1999; 12: 277-85

9. Calle-Pascual AL, Garcia-Honduvilla J, Martin-Alvarez PJ, Calle JR, Marañes JP. Influence of 16-week monotherapy with acarbose on cardiovascular risk factors in obese subjects with non-insulin-dependent diabetes mellitus: A controlled, double-blind comparison study with placebo. Diab Metabolism 1996; 22: 201-202.

10. López-Alvarenga JC, Aguilar-Salinas CA, Velasco-Pérez ML, AritaMelzer O, Guillen LE, Wong B, Brito G, Mercado V, Gómez-Pérez FJ, Rull-Rodrigo JA. Acarbose vs. bedtime NPH insulin in the treatment of secondary failures to sulphonylurea-metformin therapy in type 2 diabetes mellitus. Diabetes Obes Metab 1999; 1: 29-35.

11. Calle-Pascual AL, García-Honduvilla J, Martín-Alvarez PJ, Vara E, Calle JR, Munguira ME, Marañes JP. Comparison between acarbose, metformin and insulin treatment in type 2 diabetic patients with secondary failure to sulfonylurea treatment. Diab Metabolism 1995; 21: 256260.

12. Willms B, Ruge D. Comparison of acarbose and metformin in patients with Type 2 diabetes mellitus insufficiently controlled with diet and sulphonylureas: a randomized, placebo-controlled study. Diabet Med 1999; 16: 755-61.

13. Meneilly GS, Ryan EA, Radziuk J, Lau DC, Yale JF, Morais J, Chiasson JL,Rabasa-Lhoret R, Maheux P, Tessier D, Wolever T, Josse RG, Elahi D. Effect of acarbose on insulin sensitivity in elderly patients with diabetes. Diabetes Care 2000; 23: 1162-7.

14. Standl E, Baumgartl HJ, Fuchtenbusch M, Stemplinger J. Effect of acarbose on additional insulin therapy in type 2 diabetic patients with late failure of sulphonylurea therapy. Diabetes Obes Metab 1999; 1: 215-20.

15. Guvener N, Gedik O. Effects of combination of insulin and acarbose compared with insulin and gliclazide in type 2 diabetic patients. Acta Diabetol 1999; 36: 93-7.

16. Gentile S, Turco S, Guarino G, Oliviero B, Annunziata S, Cozzolino D, Sasso FC, Turco A, Salvatore T, Torella R. Effect of treatment with acarbose and insulin in patients with non-insulin-dependent diabetes mellitus associated with non-alcoholic liver cirrhosis. Diabetes Obes Metab 2001; 3: 33-40.

17. Holman RR, Cull AC and Turner RC on behalf on UKPDS Study Group (UKPDS 44). A randomized double-blind trial of acarbose in type 2 diabetes shows improved glycemic control over 3 years. Diabetes Care 1999; 22: 960-964.

18. Koch HH, Wudy A, Eberlein G, Quast C. Use of acarbose for eliminating the interval between meal consumption and insulin injection in patients with Type 1 diabetes. Diabetes Nutr Metab 1999; 12: 195-201.

19. Juntti-Berggren L, Pigon J, Hellstrom P, Holst JJ, Efendic S. Influence of acarbose on post-prandial insulin requirements in patients with Type 1 diabetes. Diabetes Nutr Metab 2000; 13: 7-12. 